\title{
La tomographie optique diffuse
}

\author{
Jean-Michel TUALLE, Kinia BARJEAN, Dominique ETTORI, Éric TINET \\ Laboratoire de Physique des Lasers, CNRS UMR 7538, Université Paris 13 \\ tualle@univ-paris13.fr
}

Les méthodes optiques présentent de nombreux avantages pour le diagnostic médical. Elles permettent d'accéder à des informations essentielles sur les tissus telles que leur oxygénation (par spectroscopie) ou leur perfusion (par analyse des corrélations du speckle optique). Elles demandent en revanche la résolution de problèmes inverses complexes, résolution grâce à laquelle les mesures résolues en temps peuvent se révéler un atout. De nouveaux circuits intégrés à pixels « intelligents » pourraient par ailleurs faciliter l'analyse statistique en temps réel du speckle.

\section{La tomographie optique diffuse}

Les méthodes de diagnostic optique présentent un grand potentiel pour la médecine : radiations non ionisantes, elles ouvrent la voie à des méthodes non invasives pourl'analyse des tissus, avec une instrumentation compacte et accessible à moindre coût. La spectroscopie proche infrarouge repose sur le fait que les tissus présentent, pour des longueurs d'onde situées dans l'infrarouge proche, une fenêtre de transmission avec un coefficient d'absorption particulièrement faible. En fait, c'est essentiellement l'hémoglobine, sous ses formes oxygénée et désoxygénée, qui va absorber la lumière à ces longueurs d'onde, permettant un dosage optique de cette molécule d'intérêt vital.

Une difficulté réside cependant dans le fait que les tissus sont également très diffusants à ces longueurs d'onde. Ainsi, pour traverser quelques centimètres de tissus, la lumière devra en fait parcourir des dizaines de centimètres, ce qui correspond à des temps de parcours de l'ordre de quelques nanosecondes (figure 1). La faible absorption des tissus permettra tout de même d'observer un signal significatif à travers un sein par exemple, d'où des applications potentielles en mammographie. La principale difficulté soulevée par la propagation diffuse est que cette dernière va brouiller très efficacement toute l'information spatiale sur le milieu

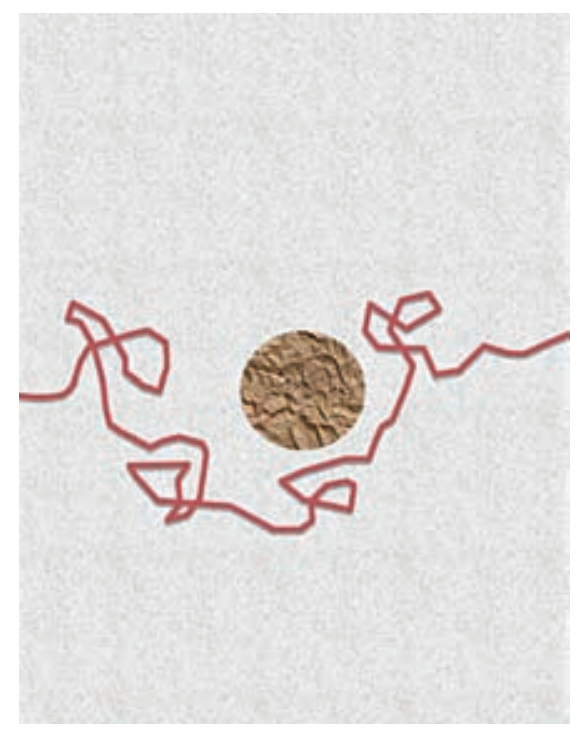

Figure 1. Pour aller d'un pointà un autre, la lumière diffuse doit parcourir des trajectoires beaucoup plus longues que la distance entre ces deux points. La lumière diffuse peut ainsi contourner les objets et brouiller l'information spatiale sur le milieu.

sondé, la lumière pouvant contourner la zone que l'on chercher à imager (figure 1).

L'objectif de la tomographie optique diffuse est de reconstruire les propriétés optiques d'un milieu diffusant, à savoir ses coefficients d'absorption et de diffusion, pour récupérer cette information spatiale et obtenir une information quantitative sur le tissu. Le coefficient d'absorption peut renseigner sur l'oxygénation du tissu, et repérer par exemple des tumeurs cancéreuses, dont le métabolisme est très différent de celui des tissus sains. Mais le coefficient de diffusion peut également être source d'information puisqu'il est relié à la structure des tissus. II peut par exemple permettre de différencier une tumeur cancéreuse d'un kyste. Pour obtenir ces informations il faut donc résoudre un problème inverse, et il s'agit là d'un défi qui occupe de nombreuses équipes.

\section{Les mesures résolues en temps}

Pour tenter de résoudre le problème inverse, il peut être utile d'augmenter le nombre de données disponibles en s'intéressant à la dynamique de propagation de la lumière diffuse. Le protocole typique pour effectuer des mesures résolues dans le temps consiste à illuminer le tissu avec une impulsion laser très courte et à suivre l'évolution temporelle de la lumière diffuse.

Cette information pourra être utilisée de plusieurs manières différentes. Par exemple, l'atténuation de la lumière dans un milieu absorbant suit la loi de BeerLambert, avec une atténuation exponentielle en fonction de la distance parcourue. Une telle loi peut être utilisée pour un milieu diffusant à condition d'avoir accès à la longueur réelle des «trajectoires » parcourues par la lumière, directement reliée au temps de transit. La résolution temporelle permet ainsi une détermination 
conjointe des coefficients d'absorption et de diffusion, détermination qui reste plus délicate sans ces données dynamiques même avec un milieu relativement uniforme.

De manière générale, les mesures résolues en temps vont permettre d'éviter le besoin de mesures absolues de l'énergie diffusée, avec tous les problèmes de calibrage que pose ce type de mesures et tous les artéfacts liés à l'état de la surface du milieu étudié. En sélectionnant les temps de transit longs sur des mesures effectuées en réflectance, on pourra même analyser un milieu en profondeur et obtenir des informations sur un organe tel que le cer- veau ou le muscle, en limitant l'influence des couches de tissus qui le recouvrent. A contrario, la sélection des temps de transit courts sur des mesures effectuées en transmittance permettra d'améliorer la résolution spatiale d'une imagerie en transillumination : la lumière ayant moins de temps pour s'écarter de l'axe optique, la zone explorée par la lumière diffuse sera en effet restreinte (figure 2).

Les échelles de temps en jeu ici étant de l'ordre de la nanoseconde, une résolution temporelle de l'ordre de quelques dizaines de picosecondes est nécessaire. Ceci implique des technologies relativement délicates et coûteuses, d'autant que
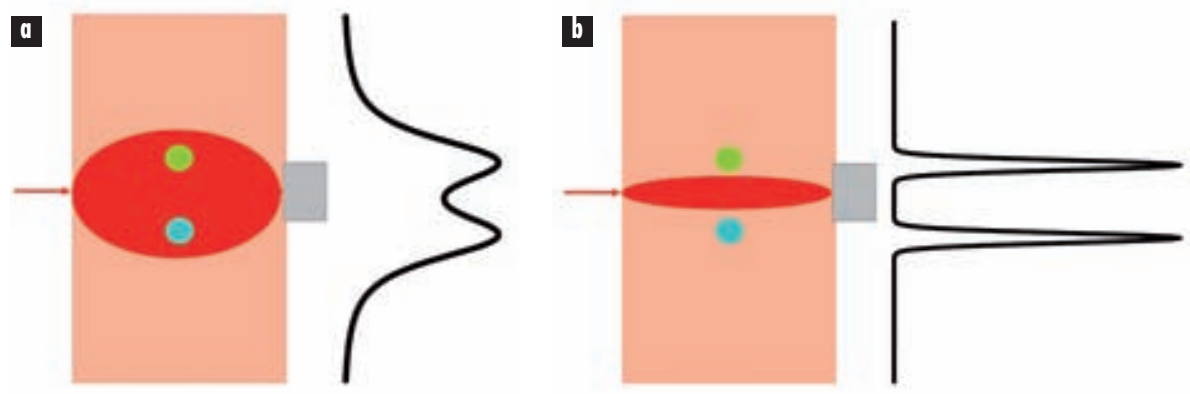

Figure 2. La lumière diffuse explore une large portion du milieu (zone rouge sur les figures $2 a$ et $2 b$ ), qui va dégrader (2a) la résolution spatiale de l'imagerie en transillumination (convolution de la zone explorée par la taille des objets). La sélection des temps courts va concentrer cette zone explorée par la lumière autour de l'axe optique, et améliorer la résolution spatiale (2b).

le niveau du signalà mesurer reste relativement faible. Une solution généralement utilisée consiste à utiliser des diodes lasers picosecondes pour l'émission de l'impulsion lumineuse, et un système de comptage de photons associéà un convertisseur temps-amplitude pour la détection résolue en temps.

\section{Mesure interférométrique de la lumière diffuse}

Une méthode alternative pour effectuer des mesures résolues dans le temps repose sur une détection interférométrique de la lumière diffuse. On considère un interféromètre avec une différence de marche non nulle entre ses deux bras (figure 3), et on balaye la longueur d'onde de la source laser monochromatique. Les champs que l'on va superposer n'auront pas exactement la même pulsation à cause de ce balayage et de la différence de temps de parcours entre les deux bras de l'interféromètre : on aura donc un battement hétérodyne du signal d'interférence, dont la fréquence sera proportionnelle à cette différence de temps de parcours.

Il est ainsi possible de remonter à la distribution des temps de parcours par l'analogue d'une transformation de Fourier ou de la détection synchrone d'un signal.

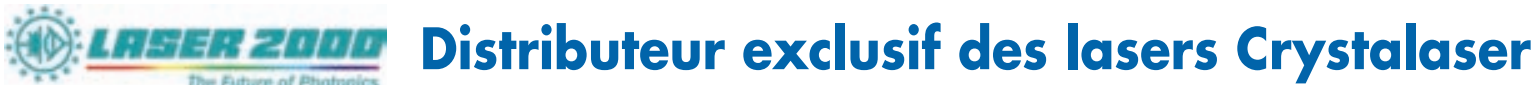

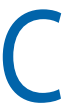
rystaLaser conçoit et développe des sources lasers à solide pompés par diode continus et Qswitchés, et intègre des diodes lasers dans des modules collimatés pour couvrir une très large gamme de longueurs d'ondes. Ces lasers ultra-compacts sont caractérisés par une très grande stabilité et fiabilité, un rendement supérieur et une excellente qualité de faisceau.

Nous vous proposons des longueurs d'onde de l'UV, à partir de $262 \mathrm{~nm}$ jusqu'à I'IR à $1444 \mathrm{~nm}$ avec des puissances de $1 \mathrm{~mW}$ à plusieurs W en fonction des modèles.

Lasers continus violets, bleus, verts et jaunes

\begin{tabular}{|c|c|c|c|c|c|c|c|c|c|c|c|c|c|}
\hline Longueur d'onde $(\mathrm{nm})$ & 375 & 405 & 430 & 445 & 473 & 488 & 523 & 527 & 532 & 542 & 555 & 561 & 593 \\
\hline Puissance $\max (\mathrm{mW})$ & 100 & 1000 & 15 & 500 & 150 & 50 & 300 & 300 & 500 & 200 & 200 & 200 & 50 \\
\hline \multicolumn{14}{|c|}{ Lasers continus rouges TEMOO } \\
\hline Longueur d'onde $(\mathrm{nm})$ & 635 & 642 & 655 & 656 & 660 & 671 & 690 & 785 & 808 & 830 & 852 & 914 & 980 \\
\hline Puissance $\max (\mathrm{mW})$ & 40 & 100 & 70 & 200 & 200 & 200 & 50 & 120 & 120 & 100 & 100 & 100 & 1000 \\
\hline \multicolumn{14}{|c|}{ Lasers continus IR } \\
\hline Longueur d'onde $(\mathrm{nm})$ & $\begin{array}{l}780 \\
990\end{array}$ & 9461 & 1030 & 1047 & 1053 & 1064 & 1122 & 1313 & 1319 & 1338 & 1342 & 1444 & 1550 \\
\hline N) & 100 & 500 & 200 & 2000 & 2000 & 3000 & 1000 & 1000 & 1000 & 1000 & 1500 & 500 & 1000 \\
\hline
\end{tabular}

Les lasers CrystaLaser sont utilisés entre autres dans des applications de spectroscopie Raman, de fluorescence, de microscopie, d'holographie, d'interférométrie aussi bien dans les domaines scientifiques qu'industriels. Des intégrations sur mesure de plusieurs lasers dans un même rack sont disponibles en fonction de votre cahier des charges.

Un large choix d'options est disponible : faisceau monomode longitudinal (longueur de cohérence de $400 \mathrm{~m}$ ), couplage sur fibre monomode et multimode, stabilité de faisceau optimisé (jusqu'à $0.25 \% \mathrm{rms})$, modulation TTL et analogique.

Lasers impulsionnels UV, bleus et verts

\begin{tabular}{|c|c|c|c|c|c|c|c|c|c|c|c|c|}
\hline Longueur d'onde $(\mathrm{nm})$ & 262 & 266 & 349 & 351 & 3554 & \begin{tabular}{|l|l|}
440 & 447 \\
\end{tabular} & 473 & 523 & 527 & 532 & 555 & 561 \\
\hline Puissance moy max (mW) & 50 & 50 & 150 & 150 & 150 & \begin{tabular}{l|l}
50 & 50
\end{tabular} & 50 & 10001 & 10001 & 1000 & 500 & 500 \\
\hline \multicolumn{13}{|c|}{ Lasers impulsionnels rouges et IR } \\
\hline Longueur d'onde $(\mathrm{nm})$ & 657 & 6606 & $671 \frac{7}{9}$ & $\begin{array}{l}750 \\
950\end{array}$ & \begin{tabular}{l|l}
946 & 104 \\
\end{tabular} & \begin{tabular}{l|l|l}
047 & 1053 \\
\end{tabular} & $1064 \mid 1$ & \begin{tabular}{l|l}
1080 & 1 \\
\end{tabular} & \begin{tabular}{|l|l|}
1313 & 1 \\
\end{tabular} & \begin{tabular}{|l|l}
1319 & 1 \\
\end{tabular} & 1342 & 1444 \\
\hline Puissance moy max (mW) & 100 & 1005 & 500 & $50 \quad 4 C$ & $400 \quad 200$ & 0002000 & 2000 & 5005 & 5002 & \begin{tabular}{|l|l}
400 & 7
\end{tabular} & 750 & 200 \\
\hline
\end{tabular}




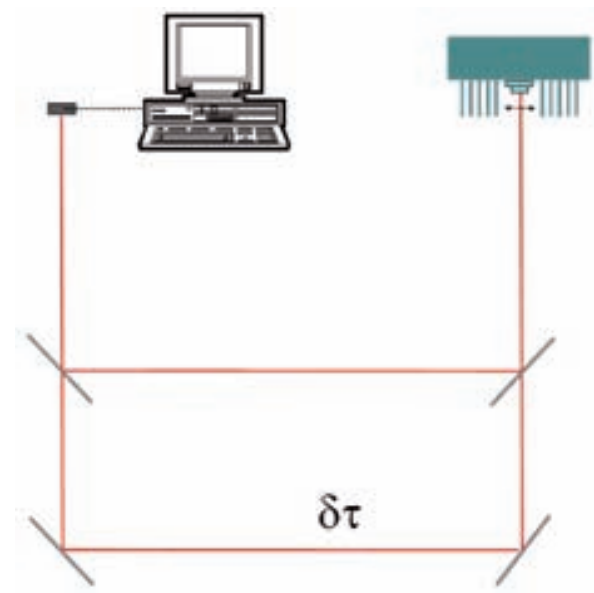

Figure 3. Principe du procédé interférométrique : les deux bras présentent une différence de temps de parcours $d t$. De par le balayage spectral de la diode laser, on aura ainsi un battement hétérodyne du signal d'interférence qu'il suffira d'analyser. Le milieu diffusant est par exemple introduit dans le bras le plus long.

La lumière diffuse, qui se présente en lumière monochromatique sous la forme d'un champ de speckle, va introduire un aléa sur ce type de données. Mais si l'on considère une quantité reliée à l'énergie du signal, comme le module carré de la composante de Fourier ou de la composante synchrone, la valeur moyenne de la quantité mesurée va exactement correspondre à l'énergie diffuse résolue dans le temps (figure 4). Il s'agit ici d'un point fondamental, l'énergie diffuse étant définie comme la moyenne d'ensemble de l'énergie électromagnétique.

L'ouverture d'un tel détecteur sera bien sûr très faible, puisque l'on observe ici un grain de speckle, mais la nature interféromètrique de cette méthode lui confère une très grande sensibilité et une grande dynamique. Il s'agit ici d'une technologie très abordable en termes de coûts et de mise en œuvre, bien adaptée pour une application clinique.

\section{Corrélations temporelles ธ en lumière diffuse}

L'étude du speckle mène à de nombreuses considérations intéressantes pour le physicien. Notamment, ses mouvements

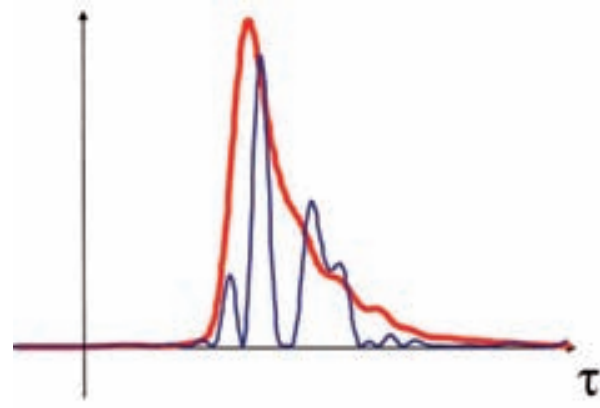

Figure 4. La lumière diffuse introduit un aléa dans le signal d'interférence (en bleu). La valeur moyenne (en rouge) représente l'énergie diffuse résolve dans le temps $(t)$.

\section{Fonction de corrélation normalisée}

En omettant la polarisation on définit la fonction de corrélation normalisée du champ électromagnétique par :

$$
g^{(1)}\left(t_{\text {corr }}\right)=\frac{\left\langle E(t) E^{*}\left(t+t_{\text {corr }}\right)\right\rangle}{\left\langle|E(t)|^{2}\right\rangle}
$$

La fonction de corrélation $\left\langle E(t) E^{*}\left(t+t_{\text {corr }}\right)\right\rangle$ se comporte comme l'énergie diffuse, mais avec un facteur d'absorption supplémentaire $\mu\left(t_{\text {corr }}\right)$. Si l'on fixe le temps de transit de la lumière à la valeurt, on aura avec la loi de Beer-Lambert pour un milieu uniforme :

$g^{(1)}\left(t_{\text {corr }}\right)=\exp \left[-\mu\left(t_{\text {corr }}\right) c \tau\right]$, cétant la vitesse de propagation dans le milieu. sont directement reliés aux mouvements microscopiques au sein du milieu. Ceci présente un intérêt direct en médecine puisque l'on peut ainsi caractériser le mouvement des globules rouges du sang et donc accéder à la perfusion du tissu. En fait, on peut montrer que les corrélations temporelles du champ électromagnétique (voir encadré) se comportent comme la lumière diffuse, mais avec un coefficient d'absorption supplémentaire.

On a ainsi un nouveau facteur de contraste directement relié à la perfusion des tissus, information très importante et complémentaire à celle apportée par l'oxymétrie. Ce nouveau contraste a amené à la notion d'imagerie d'hétérogénéité dynamique. Si l'on observe un milieu diffusant en transillumination, et que l'on introduit un objet dont les propriétés optiques sont identiques à celles du milieu, cet objet ne sera pas visible sur une simple mesure de l'énergie diffuse. Mais si ses propriétés dynamiques sont différentes (diffuseurs plus mobiles), cet objet pourra être visualisé sur des mesures de corrélations temporelles (figure 5).

Les mesures de corrélations temporelles peuvent être couplées à la méthode interférométrique de résolution tem porelle décrite précédemment. II suffit pour cela de calculer des corrélations des

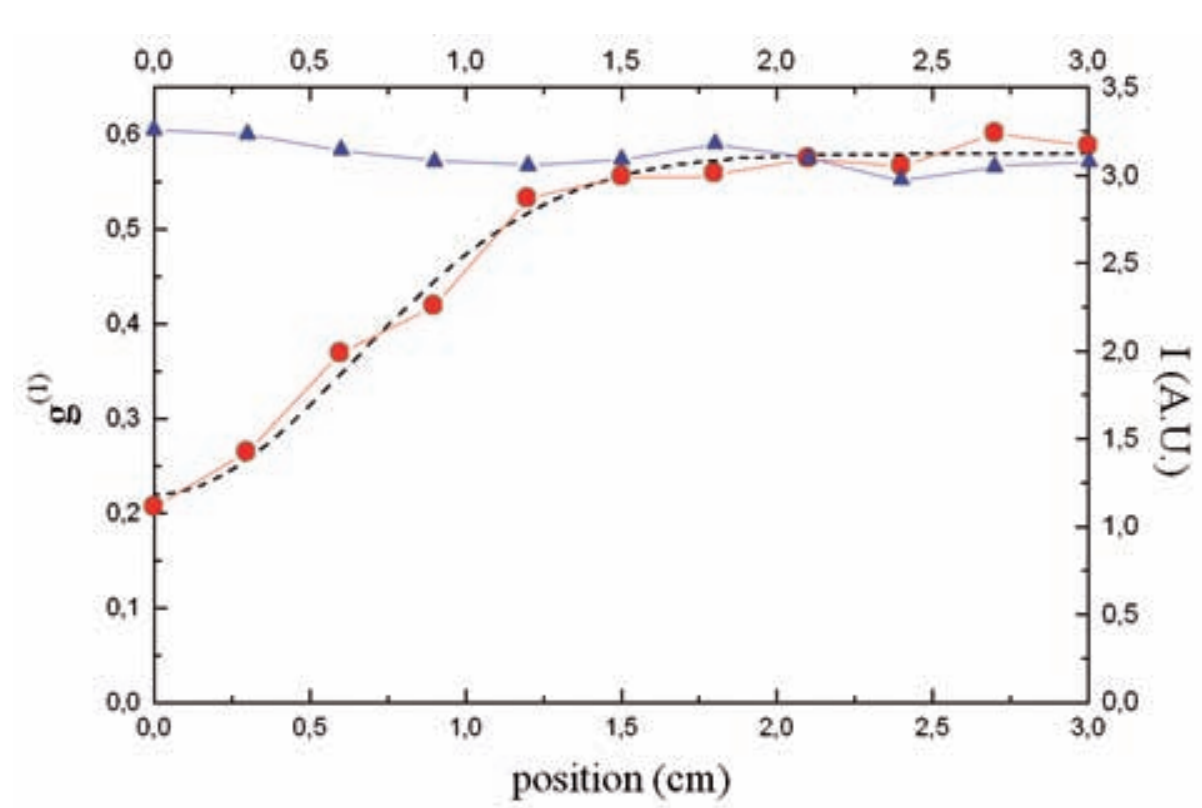

Figure 5. Imagerie d'une hétérogénéité dynamique : I'inclusion n'est pas visible sur de simples mesures de l'énergie diffuse (triangles bleus), mais elle l'est sur des mesures de corrélation (ronds rouges). 


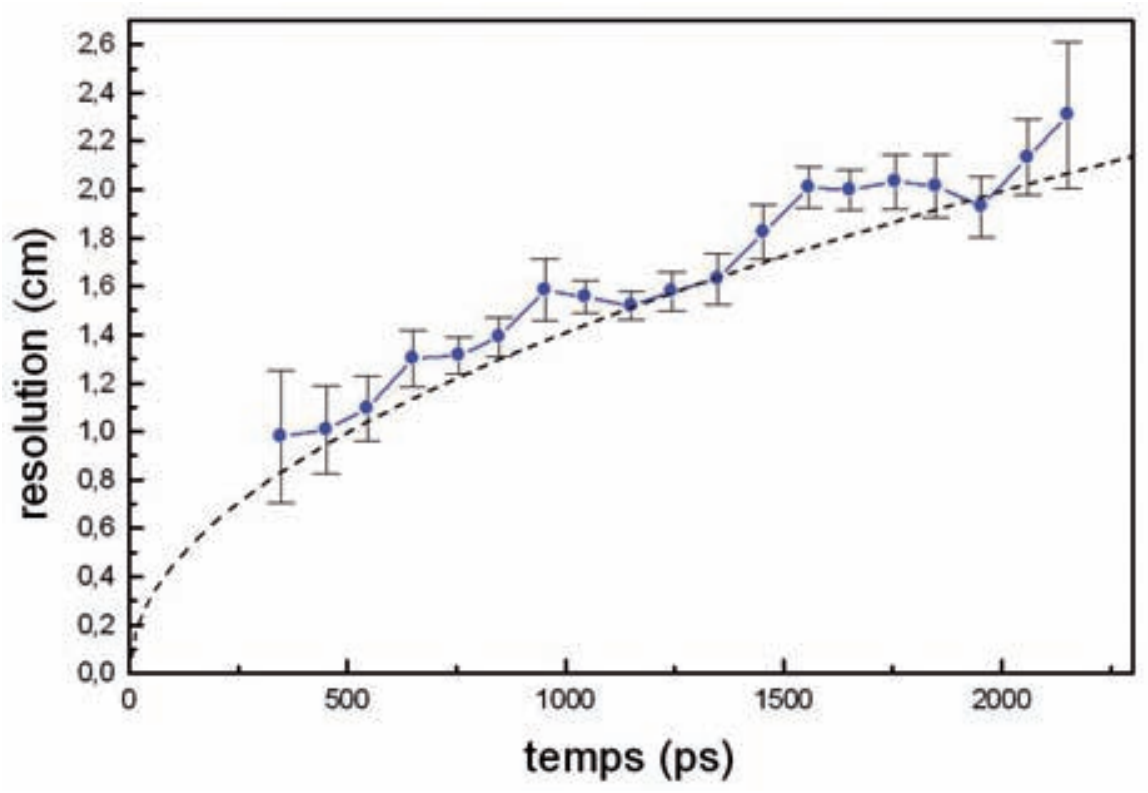

Figure 6. Amélioration de la résolution spatiale de l'image en transillumination d'une hétérogénéité dynamique avec la sélection des temps de transit courts de la lumière.

composantes synchrones au lieu du module carré. II est ainsi possible d'accéder au facteur de contraste dynamique avec en plus une sélection du temps de transit des photons. Par exemple pour l'imagerie en transillumination d'une hétérogénéité dynamique, la sélection des temps de transit courts permet d'améliorer la résolution spatiale de l'image (figure 6).

\section{Des circuits multi-pixels pour l'étude statistique du speckle}

Ces mesures interférométriques se font à l'échelle du grain de speckle. Prendre un capteur optique beaucoup plus grand qu'un grain de speckle n'apporte rien pour des raisons de cohérence spatiale (voir encadré). En revanche, il est tout à fait envisageable d'analyser simultanément plusieurs grains de speckle à l'aide d'un capteur multi-pixels.

Les caméras CCD pourraient apparaître comme une solution appropriée, bénéficiant d'un grand nombre de pixels et d'un grand savoir-faire. Il se trouve cependant que, dans le cas des tissus biologiques, le speckle bouge très rapidement, avec des temps de corrélation sub-milliseconde. Ainsi, même s'il est possible d'utiliser cette solution avec des caméras lentes ou rapides, elle ne mène pas à des performances optimales.

Par contre il est possible de développer des circuits intégrés à pixels « intelligents», capables de calculer les quantités perti-

\section{Détection interférométrique de la lumière diffuse} d'un grain de speckle. Si en revanche on réalise des mesures indépendantes au niveau de chaque grain avec un capteur multi-pixels, et que l'on somme des quantités strictement positives (module carré du signal), on obtiendra une contribution qui va croître avec le nombre de pixels.

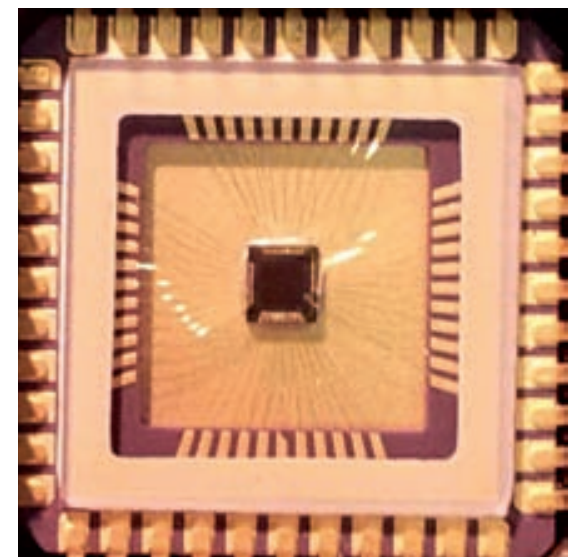

Figure 7. Circuit intégré multi-pixels pour l'analyse en temps réel d'une figure de speckle.

nentes au niveau de chaque pixel et d'en extraire les valeurs moyennes. Ce type de circuits (figure 7) permet d'atteindre de très hautes performances avec des cadences très importantes, de l'ordre de 10000 images parsecondes. Le développement dece type de capteur pourrait révolutionner ce domaine à l'avenir, permettant d'accéder à la résolution temporelle avec un matériel compact et peu coûteux.

Une détection interférométrique d'un champ électromagnétique revient non seulement à mesurer sa composante de polarisation qui coïncide avec celle du champ de référence, mais aussi à mesurer sa composante en phase avec le champ de référence. On mesure en fait une quadrature, qui est une quantité réelle et qui peut par exemple être la partie réelle du champ en notation complexe. Pour la lumière diffuse, on obtiendra une figure d'interférométrie de speckle, du type de la figure ci-dessous, avec des valeurs positives et négatives (en rouge et bleu). Ces fluctuations sont à moyenne (quasi) nulle sur un capteur grand devant la taille 